and accordingly the emitted field, will look like a sine wave starting at an extremum. The Fourier transform of this wave will contain low-frequency components responsible for terahertz emission. Unfortunately, the terahertz emission of electrons from ionization at opposite polarities of the electric field will cancel. To prevent this cancellation the trick is to use both the fundamental and the second harmonic: if the relative phase between the two is just right, the combined electric field will yield a net terahertz emission. This dependence on the relative phase was checked by varying the distance between the nonlinear crystal generating the second harmonic and the focus - the refractive-index dispersion in the air over this distance causes a change of the relative phase. Further confirmation of the proposed model comes from the measurement of the current in the plasma. This current, which is proportional to the electron velocity, induces a voltage in a wire loop near the focus. Owing to the limitations of present-day electronics, it was only possible to measure a severely low-pass filtered voltage, but this did follow the same phase dependence as the terahertz output.

Although this work marks a big step towards strong terahertz sources, as always further work is needed to improve on the present results. The most straightforward way to achieve higher terahertz pulse energies is to use higher pump pulse energies. Compared with the use of nonlinear optical crystals the present scheme has the big attraction that there is no need for concern over damage thresholds (actually, 'damaging' the atoms involved, that is ionizing them, is a requirement), so the concept can be scaled up to almost arbitrarily high pulse energies. Other possibilities include the use of pump pulses with longer wavelengths or shorter durations, or both.

Having obtained such powerful table-top terahertz sources, many new experiments that put them to use are expected in the near future. On the one hand, these sources can be used to investigate excitations in the terahertz frequency range, for example, intermolecular vibrations in molecular crystals, intra-exciton and intra-impurity transitions in semiconductors, plasmons and excitations in superconductors. On the other hand, on a short enough timescale terahertz pulses act as high d.c. fields, so that fieldinduced effects, such as nonlinear transport, at very high field strengths can be studied without damaging the sample.

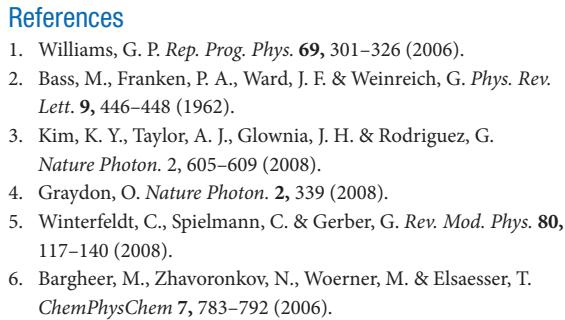

\title{
X-RAY IMAGING
}

\section{Random MADness}

X-ray crystallography has for a long time provided scientists with an insight into structure at an atomic level. As the structures become more complicated, DNA for example, image construction becomes more difficult. Taking advantage of so-called anomalous diffraction has successfully simplified this problem, but this required a periodic sample, so biological molecules had to be assembled into a crystal structure. Now, scientists from the USA, Germany and France have shown that it is possible to use anomalous diffraction to image non-periodic structures (Phys. Rev. Lett. 101, 076101; 2008).

The difficulty in applying X-ray diffraction techniques to molecules made up of thousands of atoms macromolecules - stems from the increasing number of diffracted and reflected rays. Not only the amplitudes of all of these rays need to be measured to construct a useful image, but also the phase, a technical challenge often referred to as the 'phase problem. Multiplewavelength anomalous diffraction (MAD) reduces the problem from one with many different types of atom to one that involves just one or two that scatter X-rays in an unusual way. The information gained in this way serves as a reference that provides an insight into the structure of the entire molecule.

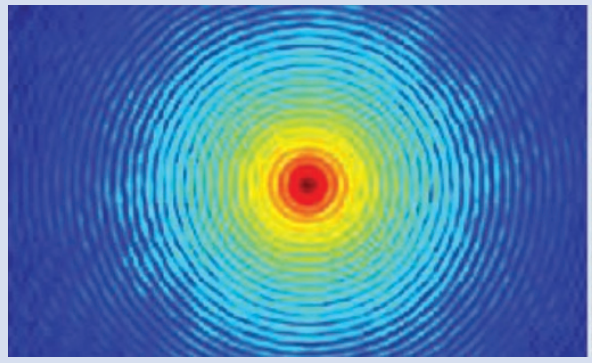

Anomalous diffraction occurs when the energy of the incident photons is resonant with an electron transition in a metallic atom, leading to the scattering of light. Anomalous scattering differs from normal scattering in that, as it is based on a resonance, it is very wavelength-dependent (but almost independent of scattering angle). By probing the sample with different wavelength X-rays, wavelength-dependent and wavelengthindependent elements of the diffraction pattern can be separated. This approach has proved a boon in structural biology, and can even be applied to macromolecules that have no metallic content, by introducing the appropriate heavy metal atoms.

Andreas Scherz and co-workers have now shown that this technique can even be used on samples that don't exhibit any periodicity. To prove this, they fabricate a test sample made up of 300-nm- and 90-nm-diameter polystyrene spheres on an aperture in a gold

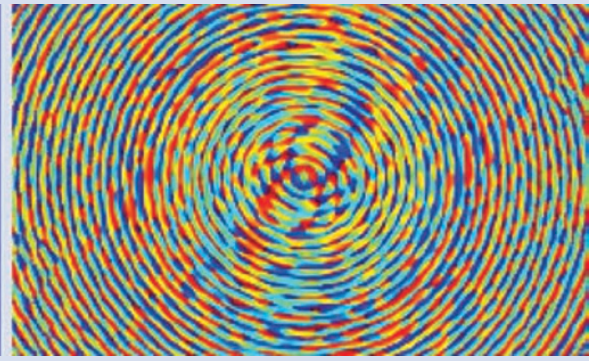

film $1.2 \mu \mathrm{m}$ across. This target was then exposed to spatially coherent X-rays (about $10^{6}$ photons $\mathrm{s}^{-1} \mu \mathrm{m}^{-2}$ ) from the Stanford Synchrotron Radiation Laboratory for between $700 \mathrm{~s}$ and 1,000 s. Two wavelengths were chosen near the edge of the ' $\mathrm{K}$ ' absorption line of carbon, one quite close to the resonance and the other a little further away (wavelengths of about $4.4 \mathrm{~nm}$ ). The interference of the diffraction patterns at these two wavelengths holds the key to creating an image. An iterative computer algorithm was used to disentangle the phase information, and in this way the team is able to image the spheres with a resolution of approximately $20 \mathrm{~nm}$.

The authors say their technique could be applied to both organic and inorganic nanostructures, enabling investigations of, for example, chemical composition or magnetic behaviour.

David Gevaux 